\title{
Residuos de desmote de algodón aglomerados: su producción y aplicación en la construcción de viviendas
}

\author{
Agglomerated cotton gin waste: its production and \\ application in housing construction
}

\author{
Romina Soledad Argento \\ J oão Ferreyra \\ Alan Murdoch Anderson \\ Maria Fernanda Carrasco \\ Ariel González \\ Ruben Marcos Grether
}

\section{Resumen}

${ }^{1}$ Romina Soledad Argento ${ }^{1}$ Universidad Tecnológica Nacional Santa Fe - Argentina

2J oão Ferreyra 2Universidad Tecnológica Nacional Santa Fe - Argentina

${ }^{3}$ Alan Murdoch Anderson ${ }^{3}$ Universidad Tecnológica Nacional Santa Fe - Argentina

${ }^{4}$ María Fernanda Carrasco 4Universidad Tecnológica Nacional Santa Fe - Argentina

${ }^{5}$ Ariel González 5Universidad Tecnológica Nacional Santa Fe - Argentina

${ }^{6}$ Ruben Marcos Grether 6Universidad Tecnológica Nacional Santa Fe - Argentina

Recebido em 07/11/17

Aceito em 28/08/18

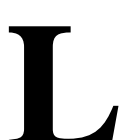

a factibilidad de producir bloques y placas mediante la aglomeración de residuos de desmote del algodón (cascarilla) con ligantes cálcicos se ha comprobado en trabajos previos. Estos residuos representan un serio inconveniente para las plantas desmotadoras, que anualmente generan gran cantidad de desechos sin destino final identificado en Argentina. La variabilidad de la producción anual de algodón, la dispersión geográfica de las desmotadoras y los costos de transporte, dificultan proponer alternativas de reutilización de alta complejidad. Se propone emplearlo como materia prima para elaborar aglomerados de cascarilla con cemento portland utilizando tecnologías sencillas, fácilmente apropiables y transferibles. Este trabajo analiza las características de los aglomerados obtenidos y la influencia que ejercen diversos parámetros de producción sobre ellas y sobre la productividad del proceso. Los resultados alcanzados muestran la mejora de la productividad del proceso mediante el empleo de aditivos de calidad industrial, así como un mejor comportamiento higrotérmico de envolventes de viviendas por la incorporación de estos aglomerados, reemplazando materiales de elevado costo y tecnologías constructivas complejas.

Palabras clave: Algodón. Desmote. Aglomerados. Mampostería. Cielorraso.

\section{Abstract}

The feasibility of producing blocks and boards through the agglomeration of cotton ginnig process waste with calcium binders was proven in previous studies. This waste represents a serious problem for ginning plants, which annually produce a high amount of waste without final destination, as is the case in Argentina. The variability of the annual cotton production, geographical dispersion of ginners and transportation costs make it difficult to propose high-complexity recycling alternatives. This study proposes that that waste is used as a raw material to manufacture composites made of cotton gin waste and portland cement, using simple, easily appropriable and transferable technologies. This paper analyses the characteristics of the composites obtained and the influence that different production parameters have on them and on the productivity of the process. The results achieved show an improvement of the process productivity by using industrial grade additives, as well as better hygrothermal behavior of housing envelopes due to the incorporation of these composites, replacing high cost materials and complex construction technologies.

Keywords: Cotton. Ginning process. Composites. Masonry. Ceiling. 


\section{Introducción}

En su primera estimación de la cosecha de 2017/18, el Comité Consultivo Internacional del Algodón (CCIA) pronostica que la producción mundial de algodón aumentará en un 2\%, respecto de la temporada 2016/17, para llegar a 23,4 millones de toneladas. Esta expansión es el resultado de un incremento en la superficie sembrada, para la cual se prevé un crecimiento de un 5\% hasta alcanzar 30,6 millones de hectáreas (REVISTA..., 2017).

En la República Argentina la producción algodonera se desarrolla principalmente en las provincias de Santa Fe, Chaco, Formosa, Santiago del Estero y Corrientes (CONSEJO..., 2013), incluyendo las tareas relacionadas con la siembra, cosecha y desmote del algodón en bruto. En las últimas décadas se registraron campañas que han alcanzado cosechas de aproximadamente 1.000.000 t de algodón en bruto, dejando atrás alrededor del $30 \%$ de su peso constituido por residuos del proceso de desmote denominados “cascarilla”, compuestos por fibrilla, cárpelos, materias extrañas, etc., sin destino previsto.

El proceso de desmote separa la fibra, que tiene el principal interés comercial, de las semillas y materias extrañas presentes en el algodón en bruto. Luego de esta separación, se seca y compacta la fibra para entregarla a la industria textil. Un análisis de los equipos desmotadores instalados en el país, indica la existencia de 164 plantas en condiciones de funcionamiento utilizando sólo el $80 \%$ de la capacidad instalada, con una capacidad nominal de desmote de 2.400 .000 t de algodón en bruto, considerando 100 días de trabajo por campaña (J.J. HINRICHSEN S.A., 1999).

Debido a que se produce una gran acumulación de estos residuos en las plantas desmotadoras, que pueden alcanzar más de un millón de metros cúbicos distribuidos en la región, en muchos casos se opta por calcinarlos originando serios problemas de polución, malestares y posibilidad de afección respiratoria, debido a que muchas desmotadoras se encuentran dentro del radio urbano (NACIONES..., 2005; EL LIBERAL, 2009).

En Argentina se afirma que el déficit habitacional ronda los 3,5 millones de hogares y, según la Fundación de Estudios para Desarrollos Inmobiliarios (FEDI), la cifra crece a un ritmo de 36 mil viviendas por año, y afecta tanto a las clases medias como a las de más bajos recursos. Según la Subsecretaría de Desarrollo Urbano y Vivienda de la Nación, se estima un déficit habitacional del $25,4 \%$ de las viviendas existentes a nivel nacional, que puede desagregarse en 2 millones de viviendas que presentan inconvenientes en la calidad de la misma, de las cuales 1,3 millones tienen situaciones de hacinamiento, y los restantes 1,5 millones son las viviendas que se necesita construir (MARTÍN, 2017).

Considerando este importante déficit habitacional que afecta a nuestro país, las deficiencias en la aislación térmica de muchas viviendas existentes (GOBIERNO..., 2001; INSTITUTO..., 2010; UNO, 1993), y a la disponibilidad de un importante volumen de estos residuos, la posibilidad de desarrollar elementos constructivos innovadores mediante la utilización de residuos de desmote del algodón, aparece como una contribución parcial a la problemática ambiental de este sector agroindustrial, al déficit habitacional de la región algodonera y a la escasez de empleo para personas sin calificación especial (PICCIONI et al., 2013).

Esta iniciativa toma en cuenta, asimismo, que el empleo de residuos agroindustriales lignocelulósicos como materia prima para la industria de la construcción, aparece como una alternativa al uso intensivo de la madera y otros recursos naturales no renovables en todo el mundo. Los científicos han obtenido buenos resultados en estas experiencias, fundamentalmente con el objetivo de dar una disposición efectiva para los desechos y reducir los impactos ambientales consecuentes. Existen antecedentes de empleo de residuos agroindustriales (trigo, azúcar, bagazo de caña, cereales, cáscaras de maíz y arroz, cáscaras de maní, fibras de coco, entre otros) en compuestos con diferentes matrices tanto cementicias como poliméricas (BARBIRATO et al., 2014; GATANI et al., 2013; GRANERO et al., 2013; JOSÉ; BERALDO, 2013; PIRAYESH; KHANJANZADEH; SALARI, 2013; VARANDAA et al., 2013; FIORELLI et al., 2012; PIRAYESH; KHAZAEIAN, 2012; PIRAYESH; KHAZAEIAN; TABARSA, 2012; MONTEIRO et al., 2011; PANYAKAEW; FOTIOS, 2011; MENDEZ; SOTELO, 2006).

De acuerdo a trabajos previos realizados (PICCIONI et al., 2013a), en los cuales las variables de análisis fueron la razón cemento/agua (C/W) y la presión de moldeo, se demostró que la variable de mayor influencia sobre las propiedades físicas y mecánicas de los materiales compuestos basados en residuos de desmote era la presión de moldeo. Además, puesto que la función del cemento (material aglomerante) es formar enlaces entre las partículas del residuo, se supone que habrá un contenido mínimo que no resultará suficiente para cubrir la superficie de estas

128 Argento, R. S.; Ferreyra, J .; Anderson, A. M.; Carrasco, M. F.; González, A.; Grether, R. M. 
partículas (y por lo tanto no permitirá que se formen enlaces fuertes entre ellas) y un máximo contenido a partir del cual la adición de más aglomerante no contribuirá a aumentar la resistencia de estas uniones, sino que formará un exceso de pasta distribuido en los aglomerados (PICCIONI et al., 2013a). Por otra parte, puede suponerse que existirá un volumen límite de vacíos entre las partículas que permitirá alojar la pasta de cemento, y que se reducirá al incrementar las presiones de moldeo.

El proceso productivo diseñado y las estimaciones de costos realizadas (PICCIONI et al., 2013b), muestran que la composición porcentual del precio de bloques para mampostería de 13 × 25 × $50 \mathrm{~cm}$, elaborados con los aglomerados desarrollados, está integrada por un $44 \%$ de materiales, $0,12 \%$ de energía, 2,3\% de amortización de equipamiento, $36 \%$ de mano de obra y $17,6 \%$ de beneficio. Estos valores revelan que el costo de este elemento constructivo depende fundamentalmente del consumo de ligante utilizado (cemento) y de la eficiencia del proceso, debido a que, a mayor productividad, la influencia de las erogaciones por mano de obra y amortización de equipamiento se reducirían significativamente.

En este sentido, se presentan las evaluaciones realizadas con el objetivo de lograr un adecuado comportamiento mecánico y térmico del material, a través de procesos sencillos de producción, que no requieran calificación especial de operarios ni impliquen consumos energéticos significativos, y se evalúa la relación entre el contenido de aglomerante y las presiones de moldeo aplicadas en la fabricación.

Asimismo, se analizan alternativas para lograr mejoras en el proceso productivo de bloques que incrementen la posibilidad de transferencia del desarrollo a los sectores productivos de la región.

\section{Materiales y métodos}

\section{Residuo de desmote (cascarilla)}

Para el desarrollo del trabajo se utilizó residuo de desmote de algodón (CA) (Figura 1) proveniente de la localidad de Villa Minetti, ubicada en la provincia de Santa Fe, Argentina. El desmote se realizó mediante la utilización del equipo Murray de 121 sierras.

La composición química del residuo de desmote, determinada de acuerdo a los métodos E 1690 (AMERICAN..., 2001), T 222 om-88, UM 250 y Wise, se resume en la Tabla 1.

\section{Granulometría}

La granulometría de estos residuos depende no solamente de las características del algodón cosechado, sino también del equipamiento de desmote. En la Tabla 2, se presentan los resultados del análisis granulométrico efectuado y en la Figura 2, se presenta la curva granulométrica correspondiente. A partir de los valores medios obtenidos se puede observar que existe preponderancia de partículas mayores a 4,75 mm, respecto al resto de los tamaños analizados, siendo lo retenido en dicho tamiz superior al 90\% y presentando un contenido de finos (pasante tamiz Nro. 80) de sólo 1,18\%.

\section{Peso por Unidad de Volumen (PUV)}

Una de las características de este residuo es su bajo peso por unidad de volumen (PUV), motivo por el cual su disposición resulta compleja y afecta grandes extensiones en los predios de las desmotadoras. Para el residuo empleado en el presente trabajo, el PUV en condición suelta alcanzó un valor de $98,87 \mathrm{~kg} / \mathrm{m}^{3}$.

Tabla 1 - Composición química del residuo de desmote

\begin{tabular}{c|c}
\hline & CONTENIDO (\%) \\
\hline Cenizas a $575{ }^{\circ} \mathrm{C}$ & 10,70 \\
Extractivos & 5,68 \\
Lignina Total & 25,53 \\
Holocelulosa & 54,53 \\
SUMATIVO & 96,24 \\
\hline
\end{tabular}

Tabla 2 - Análisis Granulométrico

\begin{tabular}{l|c}
\hline & PROMEDIO (\%) \\
\hline RETENIDO T. $\mathrm{N}^{\circ} 4$ & 95,85 \\
RETENIDO T. $\mathrm{N}^{\circ} 20$ & 1,92 \\
RETENIDO T. $\mathrm{N}^{\circ} 80$ & 1,04 \\
PASANTE T. $\mathrm{N}^{\circ} 80$ & 1,18 \\
MUESTRA FINAL & 100,00 \\
\hline
\end{tabular}


Figura 1 - Residuo de desmote de algodón en estado natural

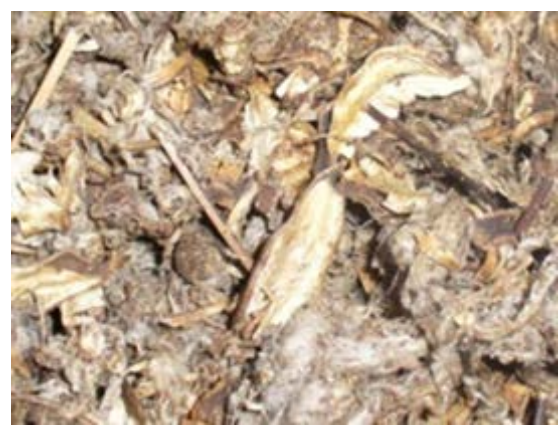

Figura 2 - Granulometría

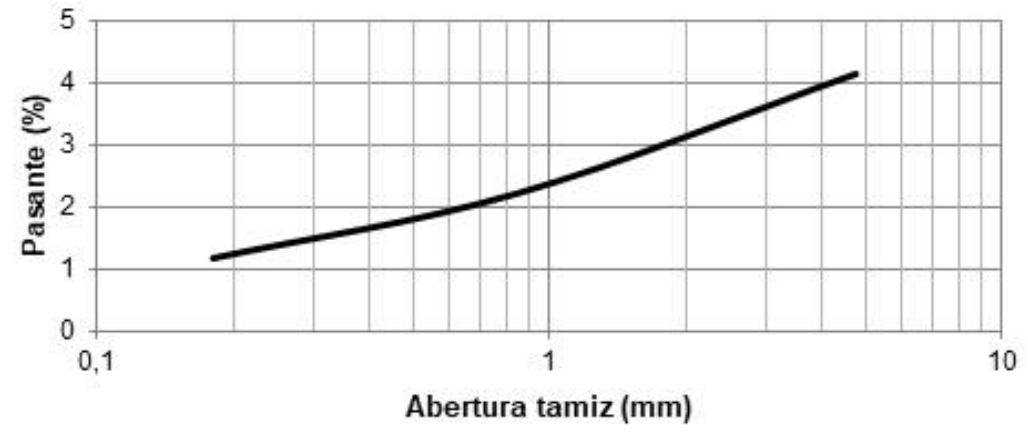

\section{Cemento Portland}

El aglomerante utilizado es cemento portland compuesto (C), con categoría resistente 40 (CPC s/ (INSTITUTO..., 2017); I (SM) s/ (AMERICAN... 2017); CPII-E s/ (ABNT, 2017)).

La composición química del cemento utilizado se describe en la Tabla 3.

\section{Aditivo acelerante de endurecimiento}

Se emplea como aditivo acelerante de endurecimiento cloruro de calcio $\left(\mathrm{CaCl}_{2} \cdot 2 \mathrm{H}_{2} \mathrm{O}\right)$ (Cc) de calidad industrial, en hojuelas con un contenido de $\mathrm{CaCl}_{2}$ del $77 \%$.

\section{Elaboración de aglomerados}

Se elaboraron aglomerados de cascarilla de desmote de algodón (CA), agua (W), cemento portland (C) y cloruro de calcio de calidad industrial (Cc). En todas las dosificaciones se utilizó una razón W/CA de 1,3g/g, razón C/W de 1,3 g/g y razón Cc/C de 0,01 g/g.

Las muestras se mantuvieron durante 24 hs en los moldes sometidas a presiones de moldeo variables entre 0.15 y $0.40 \mathrm{MPa}$, y luego se desmoldaron y mantuvieron al aire en ambiente de laboratorio (Tabla 4).

\section{Porcentaje de vacíos en el residuo compactado}

A fin de determinar la existencia de un límite superior para el nivel de presión de moldeo aplicable en la elaboración de aglomerados, se realizaron ensayos destinados a medir el contenido de vacíos presentes en el residuo a medida que se incrementa la presión a la que se lo somete.

Para esto se dispuso una masa de $90 \mathrm{~g}$ del residuo en un molde cilíndrico de 10 cm de diámetro, cuya tapa superior descendía a medida que se incrementaba la presión aplicada y que permitía medir la altura de la muestra y determinar el volumen ocupado resultante. En base a la masa conocida y al volumen medido, se determinó la densidad aparente de la muestra $\left(\mathrm{D}_{\mathrm{ap}}\right)$ para cada nivel de presión.

Paralelamente, se realizó la determinación de la densidad absoluta de las partículas que componen la cascarilla (carpelos, fibras y ramas) mediante la utilización del volumenómetro de Breuil y el procedimiento de inmersión en mercurio. Se determinaron también los porcentajes en que está presente cada fracción en el residuo, obteniendo por ponderación una densidad absoluta del residuo $\left(\mathrm{D}_{\mathrm{ab}}\right)$. 
Tabla 3 - Composición química del cemento

\begin{tabular}{c|c}
\hline COMPUESTO & PROPORCIÓN (\% EN MASA) \\
\hline $\mathrm{SiO}_{2}$ & 21,44 \\
$\mathrm{Al}_{2} \mathrm{O}_{3}$ & 4,65 \\
$\mathrm{Fe}_{2} \mathrm{O}_{3}$ & 3,10 \\
$\mathrm{CaO}$ & 57,4 \\
$\mathrm{MgO}$ & 1,30 \\
$\mathrm{SO}_{3}$ & 2,00 \\
$\mathrm{~K}_{2} \mathrm{O}$ & 0,96 \\
$\mathrm{Na}_{2} \mathrm{O}$ & 0,16 \\
$\mathrm{BaO}$ & 0,14 \\
$\mathrm{Cr}_{2} \mathrm{O}_{3}$ & $<0,01$ \\
$\mathrm{MnO}_{2}$ & 0,14 \\
$\mathrm{P}_{2} \mathrm{O}_{5}$ & 0,10 \\
$\mathrm{TiO}_{2}$ & 0,24 \\
\hline
\end{tabular}

Tabla 4 - Dosificaciones - condiciones de estudio

\begin{tabular}{c|c|c|c|c}
\hline Dosificación & $\begin{array}{c}\text { Razón W/CA } \\
\text { g/g }\end{array}$ & $\begin{array}{c}\text { Razón C/W } \\
\text { g/g }\end{array}$ & $\begin{array}{c}\text { Razón } \mathbf{C a C l}_{2} / \mathbf{C} \\
\mathbf{g} / \mathbf{g}\end{array}$ & $\begin{array}{c}\text { Presión de } \\
\text { moldeo MPa }\end{array}$ \\
\hline DA & 1,3 & 1,30 & 0,01 & 0,15 \\
DB & 1,3 & 1,30 & 0,01 & 0,24 \\
DC & 1,3 & 1,30 & 0,01 & 0,32 \\
DE & 1,3 & 1,30 & 0,01 & 0,40 \\
\hline
\end{tabular}

Finalmente, se determinó el porcentaje de vacíos presente en la muestra (V), mediante la Ecuación 1 , donde $\mathrm{V}$ es el porcentaje de vacíos en $\%, \mathrm{D}_{\mathrm{ab}}$ es la densidad absoluta del residuo en $\mathrm{g} / \mathrm{cm}^{3}$ y $\mathrm{D}_{\mathrm{ap}}$ es la densidad aparente del residuo en $\mathrm{g} / \mathrm{cm}^{3}$.

$V(\%)=1-\frac{D_{a p}}{D_{a b}}$

Los resultados se obtuvieron como promedio de 4 muestras analizadas.

\section{Caracterización de los aglomerados}

Las propiedades evaluadas para los aglomerados obtenidos fueron la densidad al momento del desmolde y para la estabilización de la masa (INSTITUTO..., 2001), la resistencia a compresión simple y el coeficiente de conductividad térmica determinado mediante el método de Less y Chorlton (MIRETTI; CITRONI; DE PAULA, 1984).

Para la determinación de la densidad se cortaron probetas prismáticas y se efectuaron medidas de largo, ancho y alto, a partir de las cuales se determinó el volumen aparente y se realizaron las determinaciones de la masa. La masa para la determinación de la densidad en el momento del desmolde fue medida inmediatamente después de retirar las muestras de los moldes, en tanto que la masa para la determinación de la densidad en estabilización se midió en el momento en que la misma registró variaciones menores al $2 \%$.
Dado que se trata de materiales no convencionales que son más deformables que los bloques de hormigón o ladrillos cerámicos tradicionales, se adoptó para la determinación de la resistencia a compresión el valor de carga correspondiente a una deformación del 10\% de la altura original del bloque. Las determinaciones se efectuaron sobre probetas prismáticas.

En el método de Less y Chorlton (MIRETTI; CITRONI; DE PAULA, 1984), para la determinación de la conductividad térmica, se coloca la muestra a ensayar entre dos placas metálicas con una aislación perimetral que evita la pérdida de calor por los laterales. Sólo la placa superior contiene una resistencia calefactora, y se determina el flujo de calor que llega a la placa inferior y el calor que esta aporta al ambiente, cuando se alcanza el estado estacionario en el sistema.

\section{Eficiencia del proceso de producción}

La posibilidad de incrementar la eficiencia de la fabricación de los aglomerados se ve condicionada fundamentalmente por el tiempo mínimo requerido de permanencia en los moldes, dado que la pasta de cemento que aglomera las partículas debe alcanzar un mínimo nivel de resistencia. Se estima que la resistencia a tracción de la pasta de cemento en el momento del desmolde debe ser igual o 
mayor a la presión de conformación del aglomerado para permitir su desmolde sin que se produzca la falla del material (Figura 3).

Una alternativa para lograr una mayor velocidad de adquisición de resistencia de la pasta de cemento es el empleo de aditivos acelerantes. El $\mathrm{CaCl}_{2}$ es uno de los aditivos más conocidos, acelera primariamente el desarrollo de la resistencia inicial del cemento debido a que actúa como un catalizador en la hidratación de las fases silicato del clinker portland (NEVILLE; BROOKS, 1998). Algunos autores indican que la incorporación de 1 a $2 \%$ de $\mathrm{CaCl}_{2}$ suele resultar suficiente para incrementar la resistencia en forma significativa a cortas edades (METHA; MONTEIRO, 1993; NEVILLE; BROOKS, 1998; SOROKA, 1979). Comercialmente se encuentra disponible, a costos muy accesibles y sin requerir medidas especiales de protección para su manipuleo, el $\mathrm{CaCl}_{2} .2 \mathrm{H}_{2} \mathrm{O}$ que contiene entre $75 \mathrm{y}$ $77 \%$ de $\mathrm{CaCl}_{2}$.

Para seleccionar las dosis $\mathrm{CaCl}_{2} \cdot 2 \mathrm{H}_{2} \mathrm{O}$ a incorporar así como los tiempos de permanencia en molde y con el fin de evaluar a los aglomerados como un sistema de variables interrelacionadas, se adoptó un diseño de experimentos central compuesto centrado (MONTGOMERY; RUNGER, 1996) que permitió evaluar la resistencia mecánica de la pasta de cemento y la estabilidad de los aglomerados mediante el análisis de superficies de respuesta en todo el dominio determinado (Figura 4).

La ecuación del modelo está dada por la Ecuación 2 (SPIEGEL; ABELLANAS, 1988):

$Y=\beta 0+\beta 1 \cdot X 1+\beta 2 \cdot X 2+\beta 3 \cdot X 1^{2}+\beta 4$.

$X 2^{2}+\beta 5 \cdot X 1 \cdot X 2$

Ec. 2

Donde:

Y: propiedad estudiada (resistencia a flexión de pastas o expansión de aglomerados);

$\mathrm{X} 1$ : porcentaje de $\mathrm{CaCl}_{2} .2 \mathrm{H}_{2} \mathrm{O}$, variable entre $1 \mathrm{a}$ $4,5 \%$ en peso de cemento (variable experimental);

$\mathrm{X} 2$ : tiempo de permanencia en molde, variable entre 4 y 24 hs (variable experimental); y

$\beta 0$ - $\beta 5$ : coeficientes estimados utilizando el método de mínimos cuadrados.

Figura 3 - Esquema de comportamiento de aglomerados

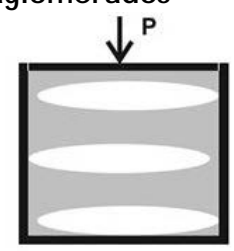

En molde

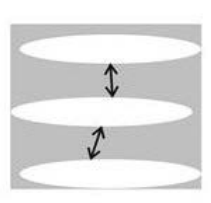

Si R pasta > P

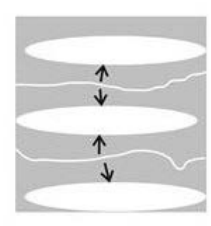

Si R pasta $<\mathrm{P}$

Figura 4 - Diseño experimental

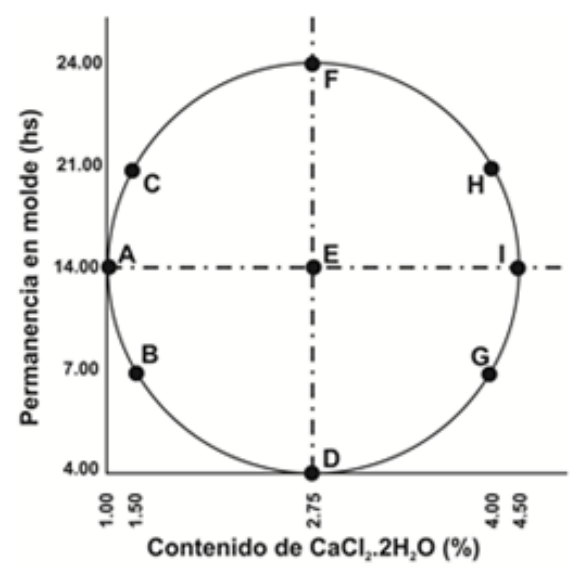

132 Argento, R. S.; Ferreyra, J .; Anderson, A. M.; Carrasco, M. F.; González, A.; Grether, R. M. 
Para evaluar la estabilidad de los aglomerados se confeccionaron bloques prismáticos con la dosificación identificada como DE (Tabla 4) (PICCIONI et al., 2013a), a partir de la cual se fue modificando el contenido de aditivo acelerante incorporado. Se midió la altura de los bloques en el centro de las 4 caras laterales inmediatamente después del desmolde y luego de transcurridas 24 hs del retiro de los moldes. En base a estas mediciones se calculó la expansión experimentada por los elementos como porcentaje de la altura registrada en el desmolde.

La adquisición de resistencia de la pasta de cemento se midió sobre pastas con razón agua/cemento de 0,77 en peso, equivalente a aquella que compone los bloques de aglomerados. Se moldearon probetas de 25 × 25 × $140 \mathrm{~mm}$ que se ensayaron a flexión con una carga centrada en la luz, inmediatamente después del desmolde. Para el ensayo a flexión se utilizó una luz de $100 \pm 1 \mathrm{~mm}$. Los resultados se obtuvieron como promedio de 4 ensayos. A pesar de que el valor de interés para la evaluación del fenómeno era la resistencia a tracción simple, se seleccionó el ensayo de resistencia a flexión debido a que el primero es muy difícil de llevar a cabo debido a las tensiones secundarias que suelen producirse en esas pruebas.

Tanto para los bloques como para las pastas, la dosis de $\mathrm{CaCl}_{2} .2 \mathrm{H}_{2} \mathrm{O}$ utilizada fue variable entre 1 a $4,5 \%$ en peso de cemento y el tiempo de permanencia en molde de 4 a 24 hs, de acuerdo a lo establecido en el diseño de experimentos.

Finalmente, sobre los bloques correspondientes a los puntos experimentales identificados como A, E e I, se realizaron ensayos de resistencia a compresión.

\section{Evaluación del comportamiento higrotérmico de envolventes}

En Argentina se establecen los valores límites de transmitancia térmica correspondientes a las condiciones de verano e invierno para muros envolventes tomando en cuenta la clasificación bioambiental del país establecida en la norma IRAM 11603 (INSTITUTO..., 2012).

Para analizar las propiedades térmicas de estos componentes constructivos se recurrió a los métodos de cálculo establecidos en la norma IRAM 11601 (INSTITUTO ARGENTINO DE NORMALIZACIÓN Y CERTIFICACIÓN, 2002) y para evaluar el riesgo de condensación superficial e intersticial se empleó la metodología indicada en la norma IRAM 11625 (INSTITUTO..., 2000).

Las comparaciones se realizan para mampostería de ladrillos comunes de 15 y $30 \mathrm{~cm}$ de espesor, mampostería de bloques de hormigón celular curado en autoclave (HCCA) de $15 \mathrm{~cm} \mathrm{y}$ mampostería de bloques intertrabados de cascarilla de $13 \mathrm{~cm}$ de espesor. En todos los casos se consideran revoques en ambas caras de acuerdo a lo recomendado por los fabricantes o por las técnicas habituales de construcción, tal como se indica en las Figuras 5a) a 5d).

En dichas figuras, las capas consideradas en la constitución de los muros son:

(a) revoque fino exterior de $0.003 \mathrm{~m}$ de espesor que consiste en un mortero calcáreo;

(b) revoque grueso exterior de $0.015 \mathrm{~m}$ que consiste en un mortero calcáreo reforzado con cemento;

(c) azotado hidrófugo de $0.006 \mathrm{~m}$ que consiste en un mortero cementicio con hidrófugo;

(d) mampostería de ladrillos cerámicos comunes;

(e) mampostería de bloques de cascarilla;

(f) mampostería de bloques de hormigón celular curado en autoclave;

(g) revoque grueso interior de $0.015 \mathrm{~m}$ que consiste en un mortero calcáreo reforzado con cemento;

(h) revoque fino interior de $0.003 \mathrm{~m}$ de espesor que consiste en un mortero calcáreo; y

(i) pintura látex interior.

Los valores de conductividad térmica y de permeabilidad al vapor de agua fueron adoptados de acuerdo a lo indicado en la norma IRAM 11601 (INSTITUTO..., 2002), a excepción de los bloques de mampostería de cascarilla para los cuales se realizaron determinaciones experimentales (PICCIONI et al., 2013a) obteniendo valores de conductividad térmica de $0,138 \mathrm{~W} / \mathrm{m}^{\circ} \mathrm{K}$ y los bloques de hormigón celular curado en autoclave (HCCA) para los cuales se adopta el valor de 0,120 $\mathrm{W} / \mathrm{m}^{\circ} \mathrm{K}$ publicado por el fabricante.

Con un objetivo similar al descripto previamente para muros, se realizaron cálculos de transmitancia térmica de acuerdo a la norma IRAM 11601 (INSTITUTO..., 2002) para diferentes estructuras de cubiertas livianas, a fin de comparar la capacidad de aislamiento térmico para condición de invierno (en la cual el flujo de calor es ascendente), y para condición de verano (en la cual el flujo de calor es descendente). 
Figura 5 - (a) mampostería de ladrillos cerámicos comunes de $15 \mathrm{~cm}$ de espesor; (b) mampostería de ladrillos cerámicos comunes de $30 \mathrm{~cm}$ de espesor; (c) mampostería de bloques de cascarilla de $13 \mathrm{~cm}$ de espesor; y (d) mampostería de bloques de hormigón celular curado en autoclave de $15 \mathrm{~cm}$ de espesor

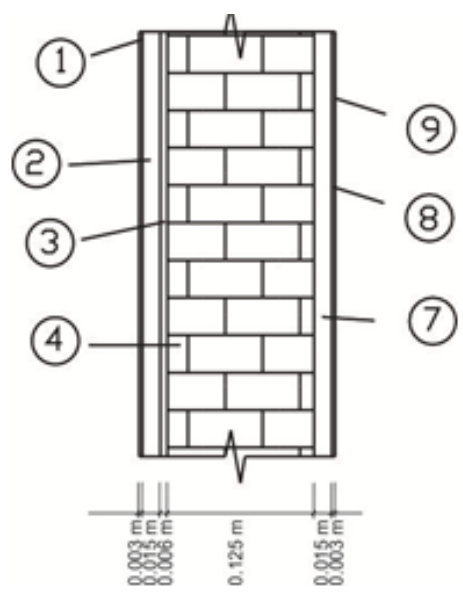

(a)

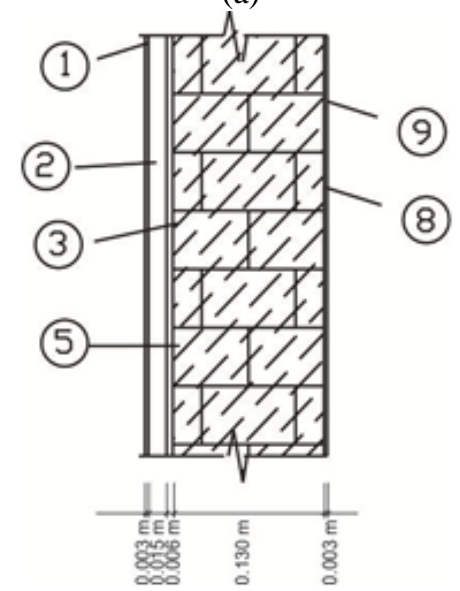

(c)

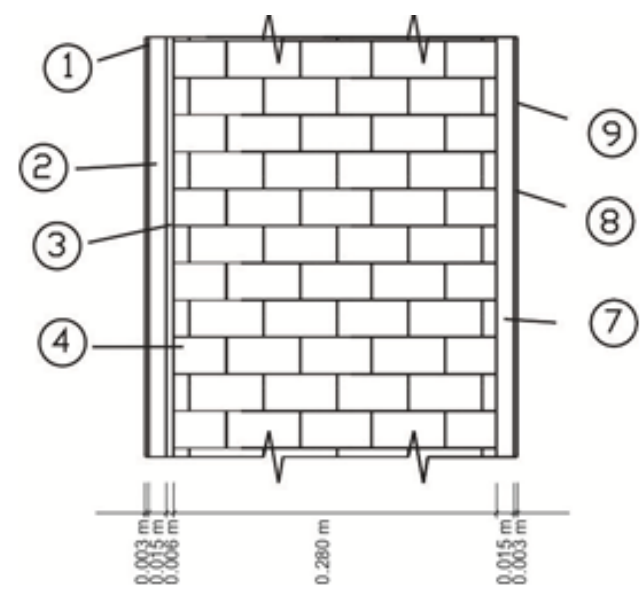

(b)

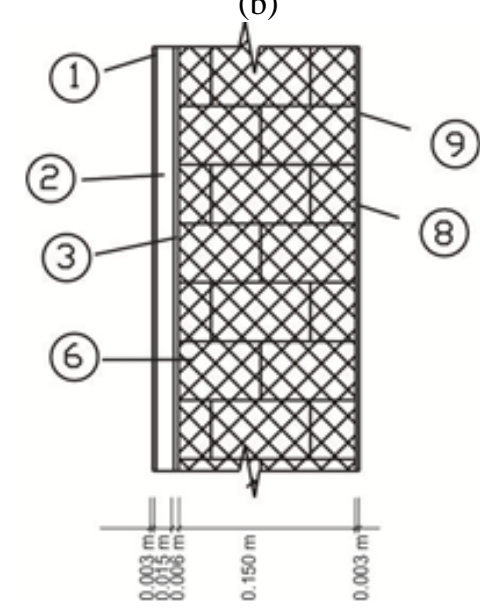

(d)

En primer lugar se llevó a cabo una tarea de relevamiento de datos para identificar las tipologías constructivas más arraigadas en la región, a fin de poder utilizarlas como parámetro para la comparación. Luego se diseñaron y modelaron los distintos paquetes constructivos y se realizaron los cálculos pertinentes.

Las estructuras de cubierta consideradas en el análisis son:

(a) cubierta de chapa sin cielorraso (Tipo A);

(b) cubierta de chapa con cielorraso sujeto a la tirantería: madera con aislante de espuma de polietileno de $10 \mathrm{~mm}$ (Tipo B), madera con aislante de lana de vidrio de $25 \mathrm{~mm}$ (Tipo C), madera con aislante de espuma de poliuretano de $25 \mathrm{~mm}$ (Tipo D), placa de cascarilla de $30 \mathrm{~mm}$ (Tipo E), placa de cascarilla de $20 \mathrm{~mm}$ (Tipo F); y

(c) cubierta de chapa con cielorraso suspendido desmontable sobre estructura metálica: placa de

yeso de $15 \mathrm{~mm}$ (Tipo G), placa de poliestireno expandido recubierta con yeso de $20 \mathrm{~mm}$ (Tipo $\mathrm{H}$ ), placa de cascarilla de $20 \mathrm{~mm}$ (Tipo I), placa de cascarilla de $30 \mathrm{~mm}$ (Tipo J).

Por otra parte, la verificación de condensación se realiza, según los procedimientos descriptos en la norma IRAM 11625 (INSTITUTO..., 2000), para determinar el riesgo de producción de condensación superficial (se produce sobre la superficie interna de la pared o techo cuando la temperatura de dicha superficie es menor que la temperatura de rocío del recinto) o condensación intersticial (se produce en el interior de las capas del muro -intersticios- o techo, debido a la disminución de su temperatura por debajo del punto de rocío). La temperatura de rocío o punto de rocío, es aquella temperatura por debajo de la cual se produce condensación para una determinada presión de vapor de agua en el ambiente o en el interior de la pared o techo. En consecuencia, la verificación consiste en 
determinar las temperaturas en la superficie y en el interior del muro y compararlas con las temperaturas de rocío en los mismos lugares.

Para efectuar estos cálculos se deben establecer como parámetros la temperatura exterior de diseño (según IRAM 11603); la humedad relativa exterior; la temperatura interior de diseño; la humedad relativa interior de diseño; la resistencia térmica superficial; los valores de permeabilidad o permeancia de los materiales; la conductividad térmica de estos materiales y los espesores considerados.

Los cálculos de las normas IRAM 11601 (INSTITUTO..., 2002) e IRAM 11625 (INSTITUTO..., 2000) están establecidos por los entes de control como indispensables para verificar las condiciones necesarias que tiene que cumplir una vivienda para alcanzar un adecuado nivel de acondicionamiento higrotérmico. Para este análisis, la norma IRAM 11605 (INSTITUTO..., 1996) fija 3 condiciones de confort: "A Recomendado”, “B - Medio” y “C - Mínimo”, para las cuales corresponden transmitancias térmicas máximas admisibles para cada zona bioambiental.
Para las zonas bioambientales Ia y Ib que corresponden a la región algodonera argentina, la norma IRAM 11605 (INSTITUTO..., 1996) establece los valores máximos admisibles de transmitancia térmica informados en la Tabla 5 para cerramientos verticales y en la Tabla 6 para cerramientos horizontales.

\section{Resultados y discusión \\ Elaboración y caracterización de los aglomerados}

Los resultados obtenidos en los ensayos de los aglomerados moldeados con las dosificaciones y presiones informados en la Tabla 4, se resumen en la Tabla 7, de acuerdo a los cuales se puede observar que la densidad se reduce desde el desmolde hasta que se alcanza la estabilización de la masa aproximadamente 20\% (18 a 22\%), variando entre 726 a $852 \mathrm{~kg} / \mathrm{m}^{3}$, resultando menor que la correspondiente a ladrillos cerámicos comunes $\left(1400\right.$ a $\left.1600 \mathrm{~kg} / \mathrm{m}^{3}\right)$ que se emplean en la zona para la construcción de muros. En las figuras 6 y 7 se pueden observar los bloques objeto de los ensayos.

Tabla 5 - Transmitancias térmicas máximas admisibles para zona bioambiental I para cerramientos verticales $\left(\mathrm{W} / \mathrm{m}^{2} \mathrm{~K}\right)$

\begin{tabular}{c|c|c|c}
\hline \multirow{2}{*}{ Condición } & \multicolumn{3}{|c}{ Nivel de confort } \\
\cline { 2 - 4 } & A & B & C \\
\hline Verano & 0,45 & 1,10 & 1,80 \\
Invierno & 0,38 & 1,00 & 1,85 \\
\hline
\end{tabular}

Tabla 6 - Transmitancias térmicas máximas admisibles según el nivel de confort para cerramientos horizontales $\left(\mathrm{W} / \mathrm{m}^{2} \mathrm{~K}\right)$

\begin{tabular}{c|c|c}
\hline Nivel de confort & Condición de Verano & Condición de Invierno \\
\hline A & 0,18 & 0,32 \\
B & 0,45 & 0,83 \\
C & 0,72 & 1,00 \\
\hline
\end{tabular}

Tabla 7 - Comportamiento de las dosificaciones evaluadas

\begin{tabular}{|c|c|c|c|c|c|c|c|c|c|}
\hline : & 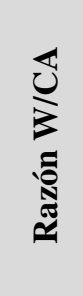 & 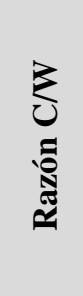 & 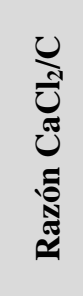 & 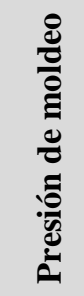 & 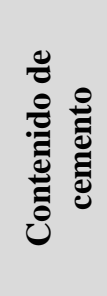 & 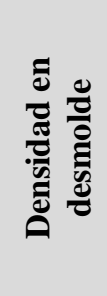 & 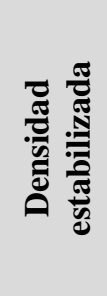 & 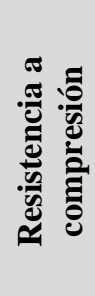 & لِّ \\
\hline & $g / g$ & $g / g$ & $g / g$ & MPa & $\mathrm{kg} / \mathrm{m}^{3}$ & $\mathrm{~kg} / \mathrm{m}^{3}$ & $\mathrm{~kg} / \mathrm{m}^{3}$ & MPa & $\mathbf{W} / \mathbf{m}^{\circ} \mathbf{K}$ \\
\hline DA & 1,30 & 1,30 & 0,01 & 0,15 & 363 & 902 & 726 & 1,03 & 0,133 \\
\hline DB & 1,30 & 1,30 & 0,01 & 0,24 & 400 & 924 & 801 & 1,01 & --- \\
\hline DC & 1,30 & 1,30 & 0,01 & 0,32 & 444 & 1034 & 827 & 1,31 & --- \\
\hline DE & 1,30 & 1,30 & 0,01 & 0,40 & 468 & 1036 & 852 & 1,55 & 0,132 \\
\hline
\end{tabular}


Figura 6 - Corte de bloques para ensayo a compresión

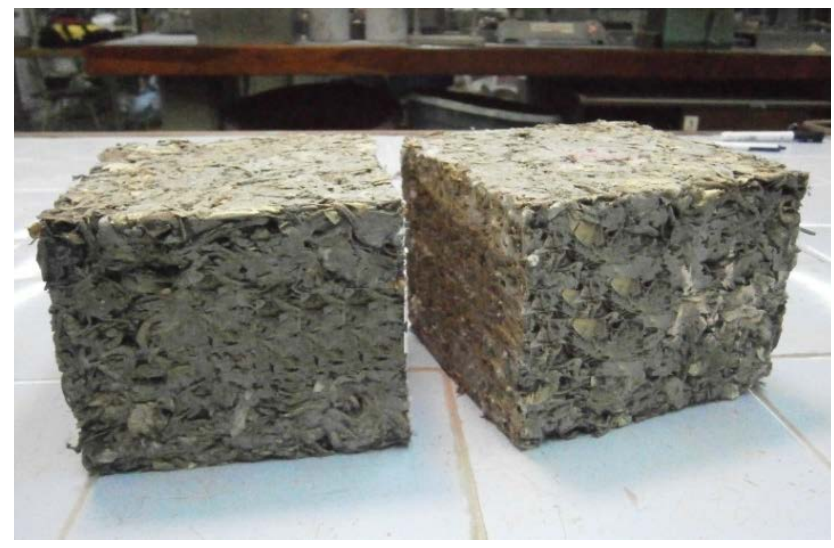

Figura 7 - Ensayo a compresión de bloques

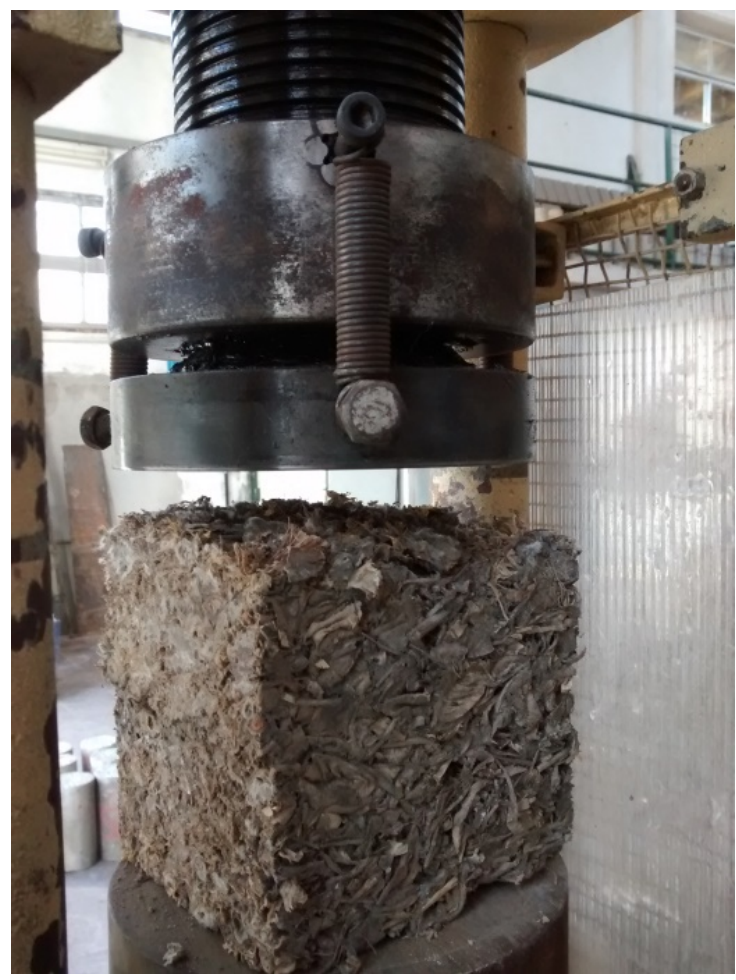

En las Figuras 8 y 9 se puede observar que a medida que se incrementa la presión de moldeo, tanto la densidad como la resistencia a compresión crecen.

Por otra parte, en la Tabla 8 se resumen los rendimientos volumétricos para cada una de las dosificaciones.

De acuerdo a la evolución de la resistencia a compresión para niveles de presión de moldeo variable, puede suponerse que al continuar incrementando la presión, la resistencia continuaría incrementándose. No obstante, se ha verificado en la práctica que existe un límite alrededor de los
0,40 MPa, a partir del cual se produce la pérdida de la lechada de cemento de los aglomerados imposibilitando su fabricación.

Este comportamiento puede explicarse a partir de la estimación del volumen que requiere la pasta de cemento y su comparación con el volumen de vacíos disponibles en el residuo a medida que se lo comprime.

La densidad aparente y los porcentajes de vacíos medidos para el residuo cuando se lo somete a niveles crecientes de presión y los volúmenes de pasta estimados para los aglomerados, se resume en la Tabla 9. 
Figura 8 - Densidad en función de la presión de moldeo

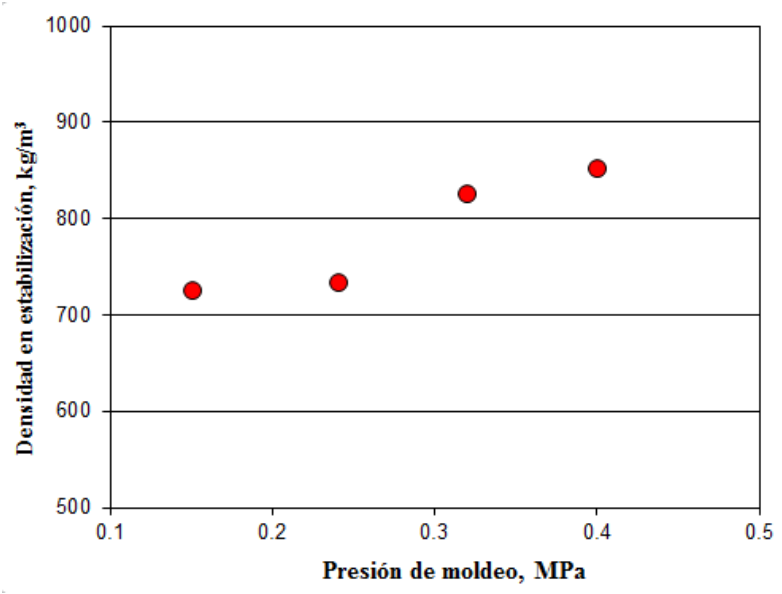

Figura 9 - Resistencia a compresión en función de la presión de moldeo

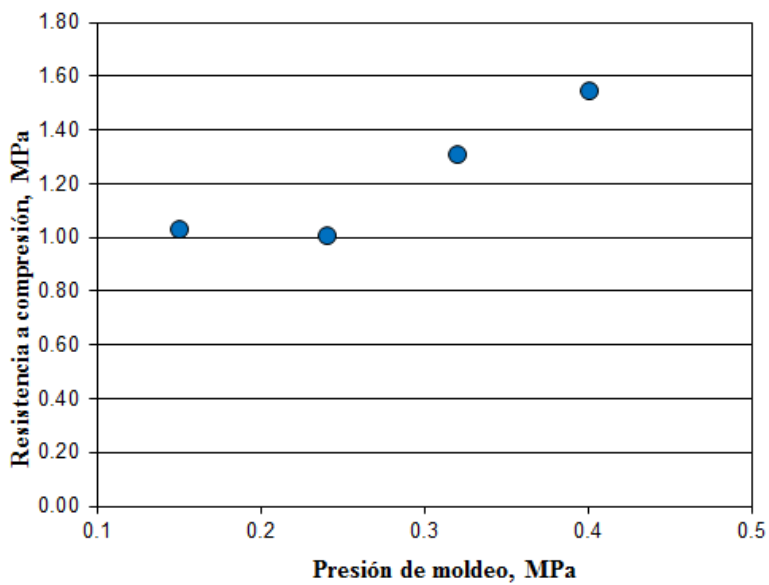

Tabla 8 - Rendimientos volumétricos para las dosificaciones evaluadas

\begin{tabular}{c|c|c|c|c}
\hline \multirow{2}{*}{ Dosificación } & Cascarilla & Cemento & Agua & $\mathbf{C a C l}_{\mathbf{2}}$ \\
\cline { 2 - 5 } & $\mathbf{k g} / \mathbf{m}^{\mathbf{3}}$ & $\mathbf{~ k g} / \mathbf{m}^{\mathbf{3}}$ & $\mathbf{~ k g} / \mathbf{m}^{\mathbf{3}}$ & $\mathbf{~ k g / \mathbf { m } ^ { \mathbf { 3 } }}$ \\
\hline DA & 215,13 & 363,57 & 279,65 & 3,71 \\
DB & 236,44 & 399,68 & 307,37 & 4,09 \\
DC & 262,61 & 443,68 & 341,35 & 4,44 \\
DE & 276,80 & 467,66 & 359,79 & 4,68 \\
\hline
\end{tabular}

Tabla 9 - Porcentaje de vacíos según nivel de presión

\begin{tabular}{c|c|c|c|c}
\hline $\begin{array}{c}\text { Presión } \\
\text { aplicada }\end{array}$ & Dap & $\mathbf{V}$ & $\begin{array}{c}\text { Volumen de vacíos en 1000 } \\
\mathbf{c m}^{\mathbf{3}} \mathbf{\text { de aglomerado }}\end{array}$ & $\begin{array}{c}\text { Volumen ocupado } \\
\text { por pasta }\end{array}$ \\
\hline MPa & $\mathbf{g} / \mathbf{c m}^{\mathbf{3}}$ & $\mathbf{\%}$ & $\mathbf{c m}^{\mathbf{3}}$ & $\mathbf{c m}^{\mathbf{3}}$ \\
\hline 0,153 & 0,15 & 74,05 & 740,50 & 409,57 \\
0,239 & 0,17 & 71,76 & 717,60 & 450,21 \\
0,320 & 0,19 & 68,45 & 684,50 & 499,81 \\
0,407 & 0,22 & 63,88 & 638,80 & 526,81 \\
0,458 & 0,23 & 62,19 & 621,90 & 559,67 \\
0,530 & 0,25 & 59,44 & 594,40 & 594,52 \\
0,649 & 0,27 & 55,23 & 552,30 & 652,12 \\
0,779 & 0,29 & 51,62 & 516,20 & 715,04 \\
0,899 & 0,31 & 48,59 & 485,90 & 773,12 \\
\hline
\end{tabular}


Puede observarse, a partir de los resultados de la Tabla 9, que al superarse un nivel de presión de moldeo de 0,458 $\mathrm{MPa}$, el volumen de vacíos disponibles en el residuo prensado no resulta suficiente para alojar la cantidad de pasta requerida para su conformación. Esta situación provoca una pérdida gradual y no uniforme de pasta desde el aglomerado, estableciendo un límite superior a los niveles de prensado utilizables.

\section{Evaluación de la eficiencia del proceso de producción}

En la Figura 10 se muestra la superficie de respuesta obtenida para la expansión de los bloques en función del contenido de $\mathrm{CaCl}_{2} \cdot 2 \mathrm{H}_{2} \mathrm{O}$ (abscisas) y del tiempo de permanencia en molde (ordenadas), medida 24 hs luego del desmolde.

Los valores de expansión registrados variaron entre 0,85\% (punto experimental F) y 16,90\% (punto experimental D). El coeficiente de correlación obtenido para el modelo de expansión fue de 0,95. Puede apreciarse que, a medida que se incrementa el contenido de $\mathrm{CaCl}_{2} \cdot 2 \mathrm{H}_{2} \mathrm{O}$ y el tiempo de permanencia en molde, la expansión tiende a reducirse. La superficie de isorrespuesta obtenida presenta una mínima curvatura, aproximándose a un plano inclinado cuya pendiente depende principalmente del contenido de $\mathrm{CaCl}_{2} .2 \mathrm{H}_{2} \mathrm{O}$ incorporado. Este comportamiento sugiere que a medida que se incrementa el contenido de $\mathrm{CaCl}_{2} .2 \mathrm{H}_{2} \mathrm{O}$ y el tiempo de permanencia en molde se alcanzan mayores niveles de resistencia en el aglomerante que permiten una mayor estabilidad de los aglomerados.

Los resultados de la Figura 11 permiten confirmar esta hipótesis ya que a medida que se incrementa el contenido de $\mathrm{CaCl}_{2} \cdot 2 \mathrm{H}_{2} \mathrm{O}$ y el tiempo de permanencia en molde (equivalente a la edad de ensayo), la resistencia a flexión de la pasta de cemento tiende a aumentar. En este caso también se observa que la superficie de isorrespuesta muestra una pendiente que obedece principalmente al contenido de $\mathrm{CaCl}_{2} \cdot 2 \mathrm{H}_{2} \mathrm{O}$ incorporado. $\mathrm{La}$ resistencia a flexión determinada en los ensayos varió entre 0,10 $\mathrm{MPa}$ (punto experimental B) y 1,05 $\mathrm{MPa}$ (punto experimental F), con un coeficiente de correlación para el modelo de resistencia a flexión de pastas de 0,98.

Al comparar las superficies de respuesta descriptas puede observarse que presentan pendientes opuestas, evidenciándose que el incremento de la resistencia permite controlar la deformación del aglomerado producido. No obstante, debe tenerse en cuenta que los ensayos de resistencia a flexión de pastas, morteros y hormigones tienden a sobrestimar el valor de resistencia a tracción del material ensayado en aproximadamente un 50\%, principalmente porque en los cálculos correspondientes se asume que la tensión varía en forma lineal a través de la sección transversal de la probeta, hecho que no es real debido a que estos materiales presentan un comportamiento tensióndeformación no lineal (METHA; MONTEIRO, 1993; SOROKA, 1979)

Tomando en cuenta estas consideraciones y los coeficientes estimados mediante mínimos cuadrados para la resistencia de las pastas, puede calcularse cuál será el menor contenido de $\mathrm{CaCl}_{2} \cdot 2 \mathrm{H}_{2} \mathrm{O}$ que permita efectuar el desmolde con seguridad a más tardar a las 8 hs del prensado. Para ello, en la fórmula correspondiente a la superficie de isorrespuesta deberá reemplazarse el valor de X2 por 8 hs e igualar la inecuación para un valor de resistencia a flexión de 0,8 $\mathrm{MPa}$, que corresponde al doble de la presión de moldeo empleada, obteniéndose como resultado una incorporación mínima de $\mathrm{CaCl}_{2} \cdot 2 \mathrm{H}_{2} \mathrm{O}$ de $4,14 \%$. Asimismo, se observa que aún para tiempos prolongados de permanencia en molde, los menores contenidos de $\mathrm{CaCl}_{2} \cdot 2 \mathrm{H}_{2} \mathrm{O}$ no permiten alcanzar un valor seguro de resistencia en la pasta, por lo cual los resultados obtenidos sugieren que la mayor incorporación de $\mathrm{CaCl}_{2} \cdot 2 \mathrm{H}_{2} \mathrm{O}$ no sólo permite acelerar los procesos de adquisición de resistencia sino que incrementa los niveles resistentes de los aglomerados (Figura 11).

Finalmente, en la Figura 12 se evalúa la influencia de la expansión de los aglomerados sobre su resistencia a compresión. Se observa que el incremento de la expansión provocado por una insuficiente resistencia de la pasta de cemento en el momento del desmolde, afecta significativamente la resistencia a compresión de los bloques. Como consecuencia de la inestabilidad de los aglomerados se produce una reducción de resistencia del orden de 0,15 MPa por cada $1 \%$ de aumento de la expansión.

En función de lo desarrollado se puede estimar que con una incorporación del orden de $4 \%$ es posible, simultáneamente, reducir el tiempo requerido de moldeo de 24 a 8 hs y mejorar el comportamiento mecánico de los aglomerados, sin modificaciones en el proceso de fabricación, sin incremento significativo del costo debido a materiales (ya que se trata de un acelerante de calidad industrial) y con una reducción significativa de la incidencia del costo de la mano de obra y de amortización de equipos. 
Figura 10 - Superficie de respuesta para expansión de bloques (\%)
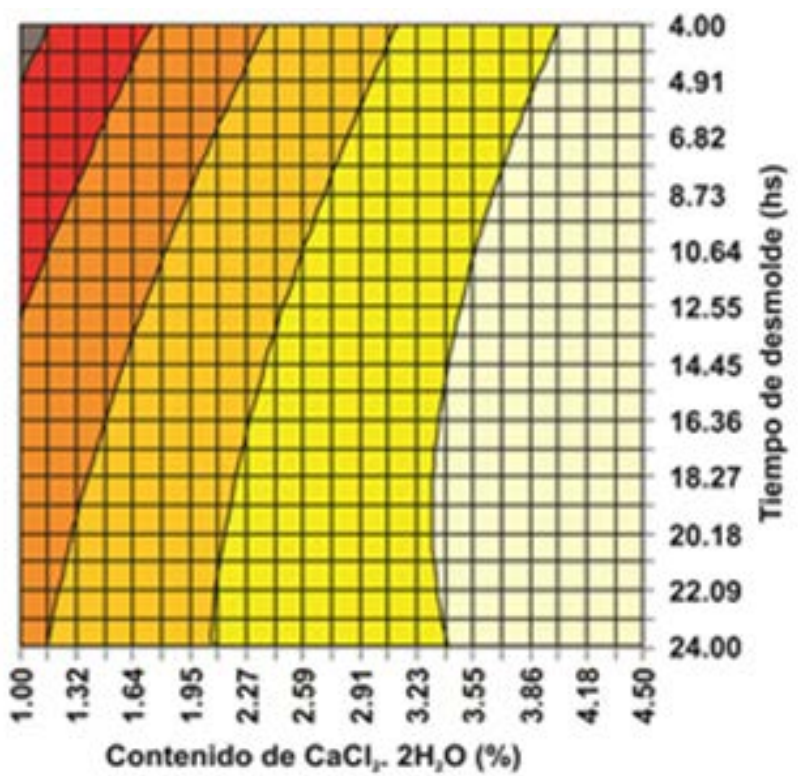

Contenido de $\mathrm{CaCl}_{x}$. 2H,

$=20.0-24.0=16.0 \cdot 20.0=12.0-16.0$
$=8.0-12.0 \quad \square 4.0 \cdot 8.0 \quad \square 0.0-4.0$

Figura 11 - Superficie de respuesta para resistencia a flexión de pastas de cemento (MPa)

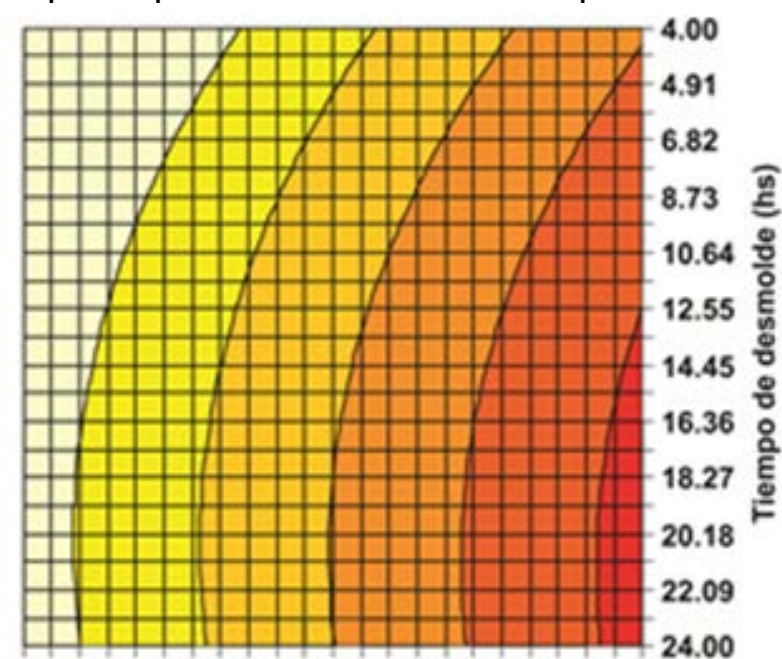

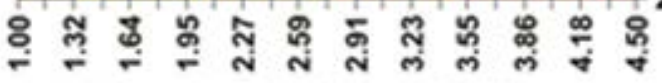

Contenido de $\mathrm{CaCl}_{2}, 2 \mathrm{H}, \mathrm{O}(\%)$

$\begin{array}{lll}=1.0 \cdot 1.2 & =0.8 \cdot 1.0 & =0.6 \cdot 0.8 \\ =0.4 \cdot 0.6 & \square 0.2 \cdot 0.4 & 0.0 \cdot 0.2\end{array}$


Figura 12 - Variación de resistencia de bloques debido a la expansión

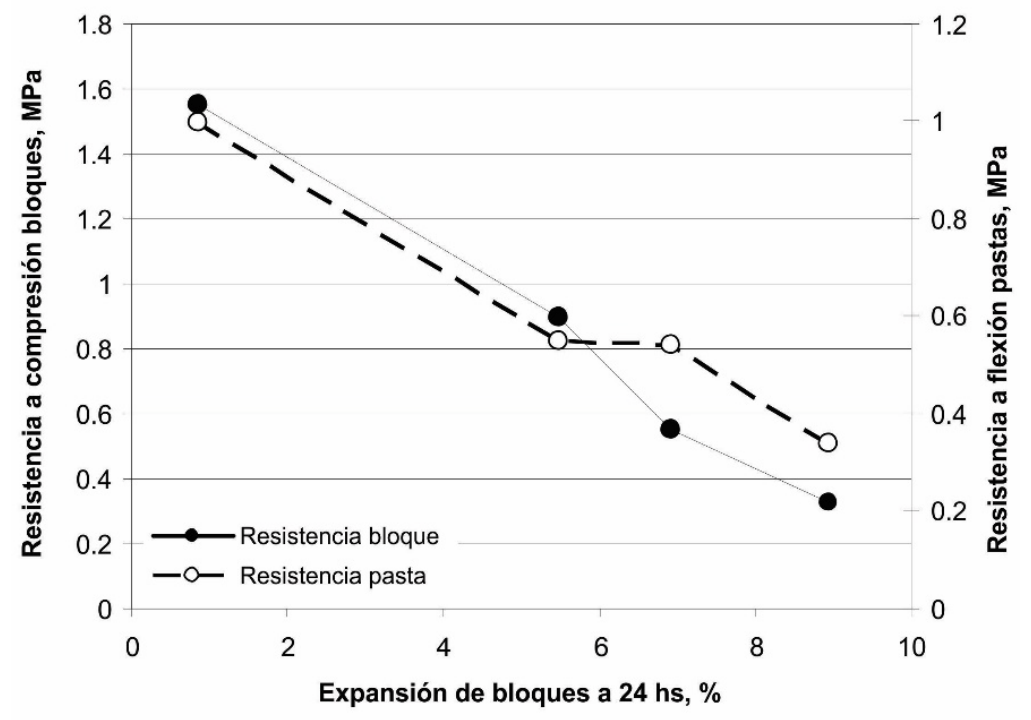

\section{Evaluación del comportamiento higrotérmico de envolventes}

En la Tabla 10 se presentan los resultados obtenidos en los cálculos de transmitancia térmica para las estructuras y materiales considerados para la construcción de muros y se comparan con los requisitos establecidos por los entes de control y la normativa para las zonas bioambientales Ia y Ib (Tabla 5).

Puede observarse, a partir de los resultados indicados en la Tabla 10, que la mampostería de ladrillos comunes de $15 \mathrm{~cm}$ de espesor no es capaz de brindar una resistencia térmica adecuada, por lo cual no alcanza a cumplir las exigencias establecidas para el mínimo nivel de confort ambiental. En el caso de incrementar el espesor hasta $30 \mathrm{~cm}$, puede alcanzarse el nivel de confort $\mathrm{C}$ solamente para la situación de verano.

Contrariamente, se observa que para la mampostería de bloques de hormigón celular curado en autoclave y de bloques de cascarilla, se cumple con las exigencias establecidas para los niveles C y B de la norma IRAM 11605 (INSTITUTO..., 1996).

En las Figuras 13(a) a 13(d) se muestran las gráficas correspondientes a la variación en las distintas capas constituyentes de los muros de la presión de vapor, la temperatura de rocío y la temperatura del muro. Puede observarse que cuando la temperatura del muro resulte menor o igual que la temperatura de rocío, se producirá condensación. En la figura 13a), puede observarse que para los muros de ladrillos comunes de $15 \mathrm{~cm}$ de espesor se verifica la producción de condensación superficial en la estación fría. La mampostería de ladrillos comunes de $30 \mathrm{~cm}$ de espesor, los muros de bloques de hormigón celular curado en autoclave y de bloques de cascarilla no presentan riesgo de condensación.

Luego de comparar los distintos paquetes tradicionales propuestos con aquellos similares que emplean placas de cielorraso de cascarilla (Tabla 11), y con los valores establecidos por los entes de control como mínimos necesarios para alcanzar el acondicionamiento térmico apropiado de una vivienda (Tabla 6), se puede observar que todos estos superan los valores máximos admisibles, no cumpliendo con los parámetros recomendados. Por otro lado, si bien la solución que ofrece el paquete Tipo D se acerca a los valores mínimos estipulados, su costo es muy elevado y su aplicación compleja, lo que dificulta su utilización en viviendas económicas. Dado que la situación Tipo A es una de las que más se repite en la zona algodonera, y siendo también la más desfavorable, se calculó el porcentaje en que se puede mejorar el aislamiento higrotérmico mediante la implementación de los distintos paquetes constructivos considerados. 
Tabla 10 - Transmitancias térmicas calculadas $\left(\mathbf{W} / \mathbf{m}^{2}{ }^{\circ} \mathrm{K}\right)$

\begin{tabular}{|c|c|c|c|c|c|c|c|}
\hline \multirow[b]{2}{*}{ Estructura } & \multirow{2}{*}{$\begin{array}{c}\text { Transmitancia } \\
\text { máxima } \\
\text { calculada } \\
\mathbf{W} / \mathbf{m}^{2}{ }^{\circ} \mathrm{K}\end{array}$} & \multicolumn{2}{|c|}{$\begin{array}{l}\text { ¿Cumple } \\
\text { Nivel C? }\end{array}$} & \multicolumn{2}{|c|}{$\begin{array}{l}\text { ¿Cumple } \\
\text { Nivel B? }\end{array}$} & \multicolumn{2}{|c|}{$\begin{array}{l}\text { ¿Cumple } \\
\text { Nivel A? }\end{array}$} \\
\hline & & 总 & 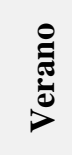 & 兽 & 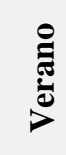 & . & 을 \\
\hline $\begin{array}{l}\text { Mampostería de ladrillos comunes de } 15 \\
\text { cm de espesor }\end{array}$ & 2,743 & NO & NO & NO & NO & NO & NO \\
\hline $\begin{array}{l}\text { Mampostería de ladrillos comunes de } 30 \\
\text { cm de espesor }\end{array}$ & 1,799 & NO & SI & NO & NO & NO & NO \\
\hline $\begin{array}{l}\text { Mampostería de bloques HCCA de } 15 \\
\text { cm de espesor }\end{array}$ & 0,693 & SI & SI & SI & SI & NO & NO \\
\hline $\begin{array}{l}\text { Mampostería de bloques de cascarilla de } \\
\qquad 13 \mathrm{~cm} \text { de espesor }\end{array}$ & 0,853 & SI & SI & SI & SI & NO & NO \\
\hline
\end{tabular}

Figura 13 - Variación de la presión de vapor, temperatura de punto de rocío y temperatura en el muro

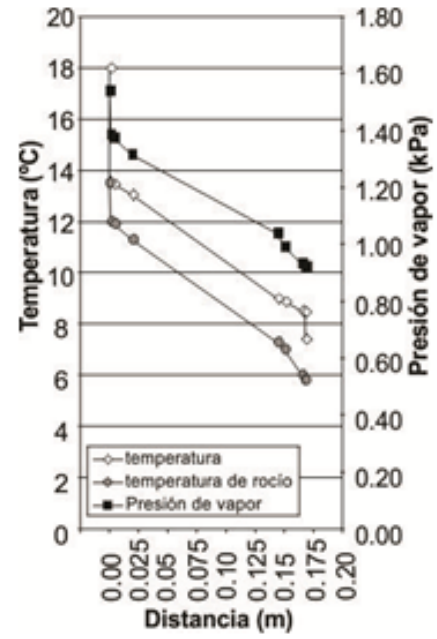

(a) Mampostería de ladrillos cerámicos comunes de $15 \mathrm{~cm}$ de espesor

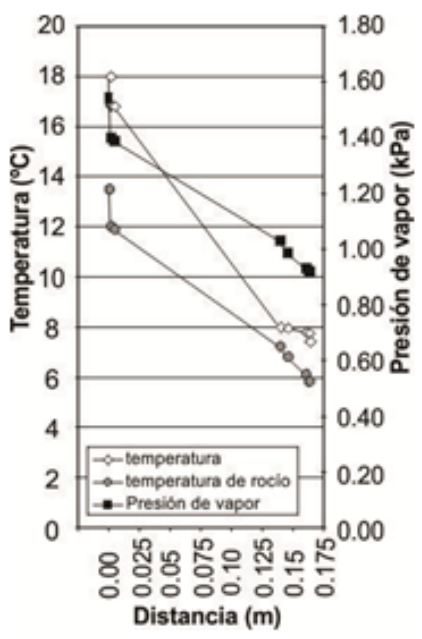

(c) Mampostería de bloques de cascarilla de $13 \mathrm{~cm}$ de espesor

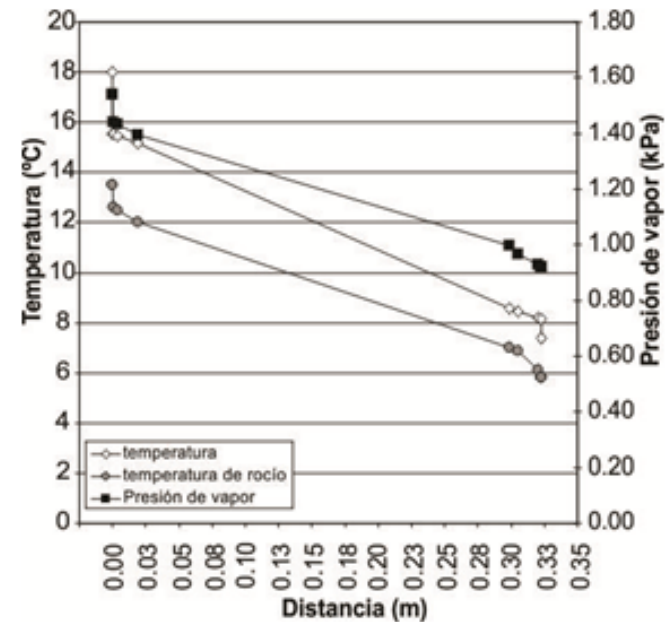

(b) Mampostería de ladrillos cerámicos comunes de $30 \mathrm{~cm}$ de espesor

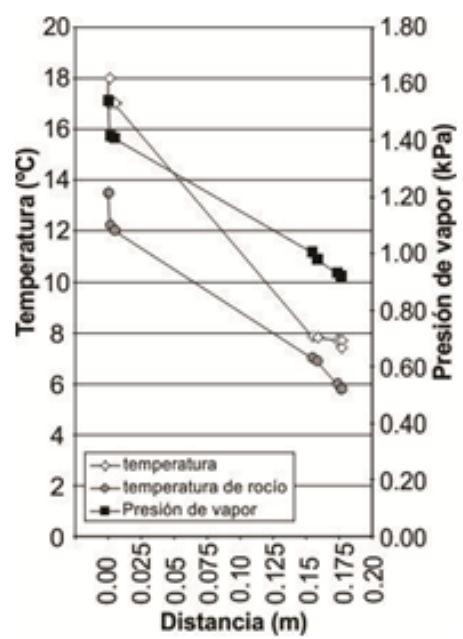

(d) Mampostería de bloques de hormigón celular curado en autoclave de $15 \mathrm{~cm}$ de espesor 
Tabla 11 - Transmitancia térmica calculada para cada paquete estructural propuesto

\begin{tabular}{|c|c|c|c|c|c|}
\hline \multirow[b]{3}{*}{ Tipo } & \multirow[b]{3}{*}{ Estructura } & \multicolumn{2}{|c|}{ Condición de Verano } & \multicolumn{2}{|c|}{ Condición de Invierno } \\
\hline & & \multirow{2}{*}{$\begin{array}{c}\mathbf{K} \\
\mathbf{W} / \mathbf{m}^{2} \mathbf{K}\end{array}$} & \multirow{2}{*}{$\begin{array}{c}\text { Mejora el } \\
\text { aislamiento } \\
\text { respecto al } \\
\text { paquete } \mathrm{A}\end{array}$} & \multirow{2}{*}{$\begin{array}{c}\mathbf{K} \\
\mathbf{W} / \mathbf{m}^{2} \mathbf{K}\end{array}$} & \multirow{2}{*}{$\begin{array}{c}\text { Mejora el } \\
\text { aislamiento } \\
\text { respecto al } \\
\text { paquete } \mathrm{A}\end{array}$} \\
\hline & & & & & \\
\hline A & $\begin{array}{c}\text { Cubierta de chapa s/tirantería } \\
\text { metálica o de madera sin } \\
\text { cielorraso. }\end{array}$ & 4,762 & - & 7,142 & - \\
\hline B & $\begin{array}{c}\text { Cubierta de chapa s/tirantería } \\
\text { metálica o de madera con } \\
\text { cielorraso de madera y } \\
\text { aislación TBA10. }\end{array}$ & 2,003 & $58 \%$ & 2,330 & $67 \%$ \\
\hline C & $\begin{array}{c}\text { Cubierta de chapa s/tirantería } \\
\text { metálica o de madera con } \\
\text { cielorraso de madera y } \\
\text { aislación lana de vidrio } 25 \\
\text { mm. } \\
\end{array}$ & 1,165 & $76 \%$ & 1,268 & $82 \%$ \\
\hline D & $\begin{array}{l}\text { Cubierta de chapa s/tirantería } \\
\text { metálica o de madera con } \\
\text { cielorraso de madera y } \\
\text { aislación poliuretano } 25 \mathrm{~mm} \text {. }\end{array}$ & 0,758 & $84 \%$ & 0,801 & $89 \%$ \\
\hline $\mathbf{E}$ & $\begin{array}{c}\text { Cubierta de chapa s/tirantería } \\
\text { metálica o de madera con } \\
\text { cielorraso de cascarilla } 30 \\
\text { mm. }\end{array}$ & 1,563 & $67 \%$ & 2,001 & $72 \%$ \\
\hline $\mathbf{F}$ & $\begin{array}{c}\text { Cubierta de chapa s/tirantería } \\
\text { metálica o de madera con } \\
\text { cielorraso de cascarilla } 20 \\
\text { mm. }\end{array}$ & 1,763 & $62 \%$ & 2,340 & $67 \%$ \\
\hline G & $\begin{array}{c}\text { Cubierta de chapa s/tirantería } \\
\text { metálica o de madera con } \\
\text { cielorraso desmontable de } \\
\text { yeso. }\end{array}$ & 2,441 & $49 \%$ & 3,443 & $52 \%$ \\
\hline $\mathbf{H}$ & $\begin{array}{c}\text { Cubierta de chapa s/tirantería } \\
\text { metálica o de madera con } \\
\text { cielorraso desmontable } \\
\text { Horpac } \AA .\end{array}$ & 1,069 & $78 \%$ & 1,225 & $83 \%$ \\
\hline $\mathbf{I}$ & $\begin{array}{l}\text { Cubierta de chapa s/tirantería } \\
\text { metálica o de madera con } \\
\text { cielorraso desmontable placa } \\
\text { cascarilla } 20 \mathrm{~mm} \text {. }\end{array}$ & 1,896 & $60 \%$ & 2,449 & $66 \%$ \\
\hline $\mathbf{J}$ & $\begin{array}{l}\text { Cubierta de chapa s/tirantería } \\
\text { metálica o de madera con } \\
\text { cielorraso desmontable placa } \\
\text { cascarilla } 30 \mathrm{~mm} \text {. }\end{array}$ & 1,667 & $65 \%$ & 2,080 & $71 \%$ \\
\hline
\end{tabular}

\section{Conclusión}

A partir de los resultados obtenidos, hasta el presente estado de avance de la investigación, se puede concluir que:

(a) es posible conformar elementos constructivos, mediante el empleo de tecnologías sencillas, a partir de la aglomeración del residuo de desmote de algodón con el cemento portland, con propiedades físicas y tecnológicas adecuadas;

(b) se pueden reducir los tiempos de moldeo requeridos para la conformación de aglomerados de residuos de desmote de algodón mediante la incorporación de contenidos variables de $\mathrm{CaCl}_{2} \cdot 2 \mathrm{H}_{2} \mathrm{O}$. Los estudios realizados permiten afirmar que la inestabilidad de estos aglomerados 
para tiempos cortos de moldeo se debe a una insuficiente resistencia de la pasta de cemento que se utiliza como ligante y que no es capaz de soportar las tensiones de tracción que se producen entre las partículas del residuo al ser liberados de la contención del molde. Con una incorporación del orden de $4 \%$ es posible reducir el tiempo requerido de moldeo de 24 a 8 hs, sin modificaciones en el proceso de fabricación ni incremento significativo del costo debido a materiales (ya que se trata de un acelerante de calidad industrial) y con una reducción significativa de la incidencia del costo de la mano de obra y de amortización de equipos;

(c) la incorporación de elementos elaborados con residuos de desmote de algodón permite mejorar el comportamiento higrotérmico de cerramientos verticales y horizontales en viviendas sin necesidad de recurrir a materiales de elevado costo ni complejizar las tipologías constructivas habitualmente empleadas en la región; y

(d) el empleo de residuos de desmote en la producción de elementos constructivos podría contribuir a paliar la problemática ambiental de este sector agroindustrial y al déficit habitacional de esta región, a la vez que podría generar alternativas laborales para la población del noreste argentino.

\section{Referencias}

AMERICAN SOCIETY FOR TESTING AND MATERIALS. ASTM E 1690: standard test method for determination of ethanol extractives in biomass. West Conshohocken, 2001.

AMERICAN SOCIETY FOR TESTING AND MATERIALS. ASTM C 595: standard specification for blended hydraulic cements. West Conshohocken, 2017.

ASSOCIAÇÃO BRASILEIRA DE NORMAS TÉCNICAS. NBR 16697: cimento portland: requisitos. Rio de Janeiro, 2017.

BARBIRATO, G. et al. Painel Aglomerado Híbrido de Casca de Amendoim Reforçado Com Partículas de Madeira Itaúba. Ciência Florestal, Santa Maria, v. 24, n. 3, p. 685-697, jul./set. 2014.

CONSEJO ARGENTINO PARA LA INFORMACIÓN Y EL DESARROLLO DE LA BIOTECNOLOGÍA. Cuaderno $\mathbf{N}^{\circ}$ 44: los cultivos transgénicos en Argentina y sus derivados. 2013. Disponible en:

$<$ http://www.porquebiotecnologia.com.ar/index.ph $\mathrm{p}$ ?action=cuaderno\&opt $=5 \& \mathrm{tipo}=1 \&$ note $=44>$. Acceso en: 18 de mayo 2011.
EL LIBERAL. Santiago del Estero: El Liberal S.A. 2009.

FIORELLI, J. et al. Particulate Composite Based on Coconut Fiber and Castor Oil Polyurethane Adhesive: an eco-efficient product. Industrial Crops and Products. Netherlands, v. 40, p. 69-75, feb. 2012.

GATANI, M. P. et al. Viabilidade Técnica de Produção e Propriedades de Painéis de Partículas de Casca de Amendoim. Revista Matéria, Rio de Janeiro, v. 18, n. 2, p. 1286-1293, jun. 2013.

GOBIERNO DE LA PROVINCIA DE SANTA FE. Censo Nacional de Población, Hogares y Vivienda. 2001. Disponible en: <http://www.santafe.gov.ar/index.php/web/content /view/full/22838>. Acceso en: 15 abr. 2011.

GRANERO, V. et al. Determinación de la Influencia del Tamaño y Forma de Partículas de Cáscaras de Maní en Paneles Aglomerados.

Quebracho, Santiago del Estero, v. 21, n. 2, p. 6780, dic. 2013.

INSTITUTO ARGENTINO DE NORMALIZACIÓN Y CERTIFICACIÓN. IRAM 11601: thermal insulation of building: calculation method: thermical properties of construction components and elements in steadystate. Buenos Aires, 2002.

INSTITUTO ARGENTINO DE NORMALIZACIÓN Y CERTIFICACIÓN. IRAM 11603: thermal conditioning of buildings: bioenvironmental classification of Republic Argentina. Buenos Aires, 2012.

INSTITUTO ARGENTINO DE NORMALIZACIÓN Y CERTIFICACIÓN.

IRAM 11605: thermal insulation of buildings: comfort conditions in buildings: maximum values of thermal transmittance for opaque enclosures. Buenos Aires, 1996.

\section{INSTITUTO ARGENTINO DE} NORMALIZACIÓN Y CERTIFICACIÓN. IRAM 11625: thermal isolation of building: verification of their hygrothermical conditions: verification of condensation risk of superficial and interstitial water vapor in the central cloths of external walls, floors and roofs of building in general. Buenos Aires, 2000.

INSTITUTO ARGENTINO DE NORMALIZACIÓN Y CERTIFICACIÓN. IRAM 9705: wood based panels: determination of density. Buenos Aires, 2001. 
INSTITUTO ARGENTINO DE NORMALIZACIÓN Y CERTIFICACIÓN.

IRAM 50000: cementos: cementos para uso general: composición y requisitos. Buenos Aires, 2017.

INSTITUTO NACIONAL DE ESTADÍSTICA Y CENSOS DE LA REPÚBLICA ARGENTINA.

Cuadro V3: total de país: viviendas particulares por material predominante de los pisos, según material predominante de la cubierta exterior del techo y presencia de cielorraso. 2010. Disponible en:

$<$ https://www.indec.gov.ar/censos_total_pais.asp?i d_tema_1 $=2 \& i d$ tema_2 $=41 \& i d \_t e m a \_3=135 \& \mathrm{t}=$ $0 \& s=0 \& c=2010>$. Acceso en: 01 de mayo 2014.

\section{J.J. HINRICHSEN S.A. Argentina: Cotton}

Ginners, Installed Capacity. Buenos Aires, 1999.

JOSÉ, F. J.; BERALDO, A. L. Tableros de Partículas de Bambú (Bambusa vulgaris Schrad) y Resina Poliuretana a Base de Aceite de Rícino (Ricinus communis L.). Ambiente Construido, Porto Alegre, v. 10, n. 4, p. 259-266, out./dez. 2010.

MARTÍN, C. La Problemática Habitacional en Argentina. 2017. Disponible en: $<$ http://www.unidiversidad.com.ar/central-laproblematica-habitacional-en-argentina >. Acceso en: 21 sep. 2017.

MENDEZ, L. I.; SOTELO, R. M. Reciclo del Marlo de Maíz: reciclado de Residuos de Construcción y Demolición (RCD) y de Residuos de Procesos (RP). In: ENCUENTRO PROCQMA, 4., San Rafael, 2006. Proceedings... San Rafael, 2006.

METHA, P. K.; MONTEIRO, P. J. M. Concrete Structure, Properties and Materials. $2^{\text {nd }}$. ed. Englewood Cliffs: Prentice Hall, Inc., 1993.

MIRETTI, R.; CITRONI, J.; DE PAULA, H. Diseño y Puesta a Punto de Un Equipo para Determinar la Conductibilidad Térmica de un Material Por el Método de Lees y Chorlton. In: REUNIÓN TÉCNICA DE LA ASOCIACIÓN ARGENTINA DE TECNOLOGÍA DEL HORMIGÓN, 6., Bahía Blanca, 1984. Proceedings... Bahía Blanca, 1984.

MONTEIRO, R. de B. F. et al. Hybrid Chipboard Panels Based on Sugarcane Bagasse, Urea Formaldehyde and Melamine Formaldehyde Resin. Industrial Crops and Products, v. 33, n. 2, p. 369-373, mar. 2011.

MONTGOMERY, D. C.; RUNGER, G. C. Probabilidad y Estadística Aplicadas a la Ingeniería. Ciudad de México D.F.: Mc Graw Hill, 1996.
NACIONES UNIDAS. Programa de las Naciones Unidas Para el Medio Ambiente. Emisiones de Dioxinas y Furanos por Quema Incontrolada de Biomasa. 2005. Disponible en:

$<$ http://webcache.googleusercontent.com/search?q =cache:hcGYTzNDHDAJ:chm.pops.int/Portals/0/ download.aspx\%3Fd\%3DUNEP-POPS-

TOOLKIT.1-

5.Spanish.PDF $+\& c d=1 \&$ hl=es\&ct=clnk\&gl=ar $>$. Acceso en: 10 abr. 2011.

NEVILLE, A. M.; BROOKS, J. J. Tecnología del Concreto. Ciudade de México D.F.: Trillas, 1998.

PANYAKAEW, S.; FOTIOS, S. New Thermal Insulation Boards Made From Coconut Husk and Bagasse. Energy and Buildings, v. 43, n. 7, p. 1732-1739, jul. 2011.

PICCIONI, J. et al. Composites Based on Cotton Gin Waste and Cement for Housing Construction. In: INTERNATIONAL CONFERENCE ON NON-CONVENTIONAL MATERIALS AND TECHNOLOGIES, 14., João Pessoa, 2013. Proceedings... João Pessoa, 2013.

PICCIONI, J. et al. Masonry Blocks Produced From Cotton Gin Trash. In: CONGRESO INTERNACIONAL DE CIENCIA Y TECNOLOGÍA DE METALURGIA Y MATERIALES; SIMPOSIO INTERNACIONAL SOBRE MATERIALES LIGNOCELULÓSICOS, 13., Puerto Iguazú, 2013. Proceedings... Puerto Iguazú, 2013a.

PICCIONI, J. et al. Propuesta de Cielorraso Constituido Por Aglomerados de Cascarilla de Algodón. In: PATOLOGÍA, CONTROL DE CALIDAD Y REHABILITACIÓN EN LA CONSTRUCCIÓN, 1., Mendoza, 2013. Proceedings... Mendoza, 2013b.

PIRAYESH, H.; KHANJANZADEH, H.; SALARI, A. Effect of Using Walnut/Almond Shells on the Physical, Mechanical Properties and Formaldehyde Emission of Particleboard.

Composites: Part B, v. 45, n. 1, p. 858-863, feb. 2013.

PIRAYESH, H.; KHAZAEIAN, A. Using Almond (Prunus amygdalus L.) Shell as a Bio-Waste Resource in Wood Based Composite. Composites: Part B, v. 43, n. 3, p. 1475-1479, apr. 2012.

PIRAYESH, H.; KHAZAEIAN, A.; TABARSA, T. The Potential for Using Walnut (Juglans regia L.) Shell as a Raw Material for Wood-Based Particleboard Manufacturing. Composites: Part B, v. 43, n. 8, p. 3276-3280, dec. 2012. 
REVISTA PARA EL SECTOR ALGODONERO. 2017. Disponible en:

<http://www.agroindustria.gob.ar/sitio/areas/algod on/informes/_archivo//830000_2017/831200_Ener o/831201_Revista\%20para\%20el\%20Sector\%20A lgodonero\%20N\%C2\%BA\%201\%20-

\%202017.pdf>. Acceso en: 15 de agosto. 2017, p. 20.

\section{SOROKA, I. Portland Cement Paste and}

Concrete. London: MacMillan Press Ltd., 1979.
SPIEGEL, M.; ABELLANAS, L. Fórmulas y Tablas de Matemática Aplicada. Madrid: Mc Graw Hill, 1988.

UNO. Santa Fe: Grupo América. 1993.

VARANDAA, L. et al. Oat Hulls as Addition to High Density Panels Production. Materials

Research, São Carlos, v. 16, n. 6, p. 1355-1361, nov./dec. 2013.

\section{Romina Soledad Argento}

Centro de Investigación y Desarrollo para la Construcción y la Vivienda, Facultad Regional Santa Fe | Universidad Tecnológica Nacional | Lavaisse 610 - Santa Fe - Argentina | S3004EWB | Tel.: (54) 93424601579 | E-mail: romina_argento@hotmail.com

J oão Ferreyra

Centro de Investigación y Desarrollo para la Construcción y la Vivienda, Facultad Regional Santa Fe | Universidad Tecnológica Nacional | E-mail: joaoferreyra7@gmail.com

\section{Alan Murdoch Anderson}

Centro de Investigación y Desarrollo para la Construcción y la Vivienda, Facultad Regional Santa Fe | Universidad Tecnológica Nacional | E-mail: alanma2590@gmail.com

\section{María Fernanda Carrasco}

Centro de Investigación y Desarrollo para la Construcción y la Vivienda, Facultad Regional Santa Fe | Universidad Tecnológica Nacional | E-mail: mcarrasc@frsf.utn.edu.ar

\section{Ariel González}

Departamento de Ingeniería Civil, Facultad Regional Santa Fe | Universidad Tecnológica Nacional | E-mail: aagonzal@frsf.utn.edu.ar

\section{Ruben Marcos Grether}

Centro de Investigación y Desarrollo para la Construcción y la Vivienda, Facultad Regional Santa Fe | Universidad Tecnológica Nacional | E-mail: rubengrether@yahoo.com.ar

\section{Revista Ambiente Construído}

Associação Nacional de Tecnologia do Ambiente Construído

Av. Osvaldo Aranha, 99 - 3o andar, Centro

$$
\begin{gathered}
\text { Porto Alegre - RS - Brasil } \\
\text { CEP 90035-190 }
\end{gathered}
$$

Telefone: +55 (51) 3308-4084

Fax: +55 (51) 3308-4054

www. seer. ufrgs. br/ ambienteconstruido

E-mail: ambienteconstruido@ufrgs.br 